\title{
Multiple Teeth Extractions, Confection and Installation of Total Prostheses in Patients with Renal Insufficiency: Case Report
}

\author{
Stephanie Albuquerque de Souza Brandão ${ }^{1 *}$, Thayse Targino Morais ${ }^{1}$, Gabriel Silveira Pinto Brandão \\ and Rafael Guedes de Paiva ${ }^{1}$ \\ ${ }^{1}$ UNIPÊ-University Center of João Pessoa, Brazil \\ ${ }^{2}$ Institute of Sciences of FUNORTE/SOEBRAS, Brazil

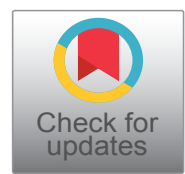

*Corresponding author: Stephanie Albuquerque de Souza Brandão, UNIPÊ-University Center of João Pessoa, Rua José Patrício de Almeida, 79/102. Jardim Oceania, João Pessoa, Paraíba, Brazil

\begin{abstract}
Patient F.A.M.S had several teeth extractions after medical authorization. To do this, forceps \#150 and \#151 were used and amoxicillin and dipyrone were prescribed. It is important to maintain oral health in patients with CKD since these are potential candidates for kidney transplantation. Due to the patient's dissatisfaction, it is indispensable, in complex cases like this, the elaboration of a term of consent, written that the patient is aware of the situation and expectation of his/her treatment, signed by the patient.
\end{abstract}

\section{Keywords}

Tooth extraction, Renal insufficiency, Denture, Complete

\section{Introduction}

Chronic Kidney Disease (CKD) is a worldwide public health problem. Its prevalence is over $10 \%$ in many countries, and the number is rising at an alarming rate. While the main risk factors for CKD are hypertension, diabetes, and obesity, periodontal disease has also been proposed as a non-traditional risk factor. CKD or its treatment may affect salivary flow rate and cause hyposalivation and thus increase the susceptibility to symptoms and discomfort such as xerostomia, dysphagia, (difficulty of swallowing), dysgeusia (an alteration in taste sensation), and Burning Mouth Sensation (BMS). Hyposalivation also predisposes patients to oral and dental problems such as caries, periodontal disease, and Candida infections [1].

According to Costa Filho [2], the dental care must be performed in alternating with hemodialysis. When it is necessary to use antibiotic, analgesic or anti-inflammatory medication, drugs with hepatic metabolism should be used. However, whenever possible, the patient's nephrologist should be contacted for adjustment and choice of medication.

Patients with an organ transplant need immunosuppressive drugs to prevent rejection for the rest of their lives. Because of immunosuppression, these patients may develop oral lesions like gingival overgrowth induced by ciclosporin or by calcium channel blockers, or oral candidiasis, viral infections, and malignancies, such as lip cancer. Since patients are susceptible to oral lesions after organ transplantation due to their immunosuppressive or antihypertensive medication, it is important to examine these patients regularly to allow early detection of oral diseases and facilitate proper treatment to avoid major complications [3].

Although there are inconclusive outcomes regarding the prevalence of caries in hemodialysis patients, most studies describe a high incidence of periodontal disease in these patients, where poor oral hygiene with increased deposits of plaque and calculus, identifying the presence of gingival inflammation, deep periodontal pockets, clinical attachment level loss and bone loss [4].

According to Lopes [5], in dental procedures involving bleeding, acetylsalicylic acid should be avoided. It irreversibly inhibits platelet thromboxane, responsible for platelet aggregation. The use of medications

Citation: Brandão SAS, Morais TT, Brandão GSP, de Paiva RG (2018) Multiple Teeth Extractions, Confection and Installation of Total Prostheses in Patients with Renal Insufficiency: Case Report. Int J Oral Dent Health 4:073. doi.org/10.23937/2469-5734/1510073

Accepted: December 19, 2018: Published: December 21, 2018

Copyright: (C) 2018 Brandão SAS, et al. This is an open-access article distributed under the terms of the Creative Commons Attribution License, which permits unrestricted use, distribution, and reproduction in any medium, provided the original author and source are credited. 
containing aspirin and non-steroidal anti-inflammatory drugs is not advisable because they present antiplatelet action.

The presence of caries, endodontic lesions, dental and periodontal abscesses, periodontitis, pericoronitis, mucositis and peri-implantitis serves as a gateway for microorganisms into the bloodstream, leading to an increase in morbidity and mortality potential of patients with CKD undergoing hemodialysis [6].

The increased level of Gram-negative microorganisms responsible for the development and progression of periodontal inflammation increases the immunological response to bacterial antigens. This may be the link between periodontal disease and CKD due to a concomitant infection and inflammation. Studies shows that the periodontitis in CKD patients was severe, with increased frequency in the presence of perio pathogens and showed in can be, though not always, observed in this group because of the lack of interdisciplinary care for these patients. Studies suggests that the higher prevalence of periodontal diseases was mainly caused by oral hygiene negligence, rather than by chronic uremia [4].

Certain drugs that are nephrotoxic would need an altered dose, altered dosage interval, or avoided, including acyclovir, amoxicillin, NSAIDs, opioids, and tetracycline. In patients receiving hemodialysis, infective endocarditis can develop from the arteriovenous fistula. Currently, the only guidelines that address antibiotic prophylaxis for patients with CKD come from the American Heart Association (AHA), which recommends antibiotic Prophylaxis for patients undergoing hemodialysis who have known cardiac risk factors [7].

Affected patients require special care, such as dosage adjustment, or even contraindication to certain medications routinely employed in dentistry. With this, the dentist has the duty to know them and to know in which situations can prescribe them. Patients with this systemic impairment require differentiated procedures, such as prior medical and laboratory evaluation [2].

The present study aims to report on a case of confection and installation of total superior and after multiple teeth extractions in a patient with CKD.

\section{Case Report}

52-year-old patient, F.A.M.S, went to the ClinicSchool of the University Center of João Pessoa-UNIPÊ in the first week of class, passing by the screening clinic, complaining about his dental condition. There were only 9 (nine) teeth in his oral cavity, all of them with indication for extraction and subsequent prosthetic rehabilitation in both arches (Figure 1).

During the anamnesis, the patient reported that hemodialysis (with sodium heparin), 03 (three) times per week was used for the treatment of renal failure. His goal was to be rehabilitated with the total upper and lower dentures to wait for the authorization of his kidney transplant.

At the end of the first consultation, a written medical authorization was requested to enable the students and the teacher to carry out the planned dental extraction. When the patient returned with written authorization, he reported that the medication had not been placed during hemodialysis. So, his pressure was measured $(130 \times 90 \mathrm{mmHg})$ and surgery was started. The surgical planning of the first day of surgery was the extractions of the dental elements 21, 22, 53, 14 and 15 (Figure 2).

A local infiltrative anesthesia (lidocaine $2 \%$ ) was performed, syndesmotomy with Molt detachment \#9 and dislocation with apex lever. For the extraction of elements, the forceps number 150 was used. The cavity was cleaned with saline and curetted with Luca's curette.

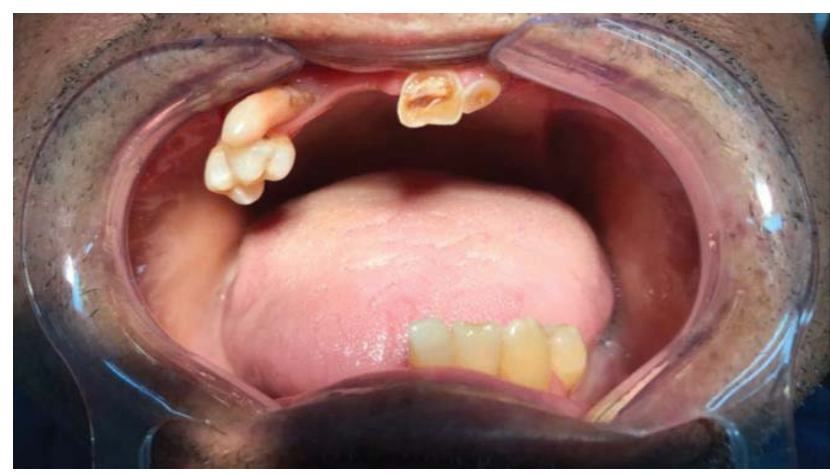

Figure 1: Dental condition: Extraction and subsequent prosthetic rehabilitation in both arches.
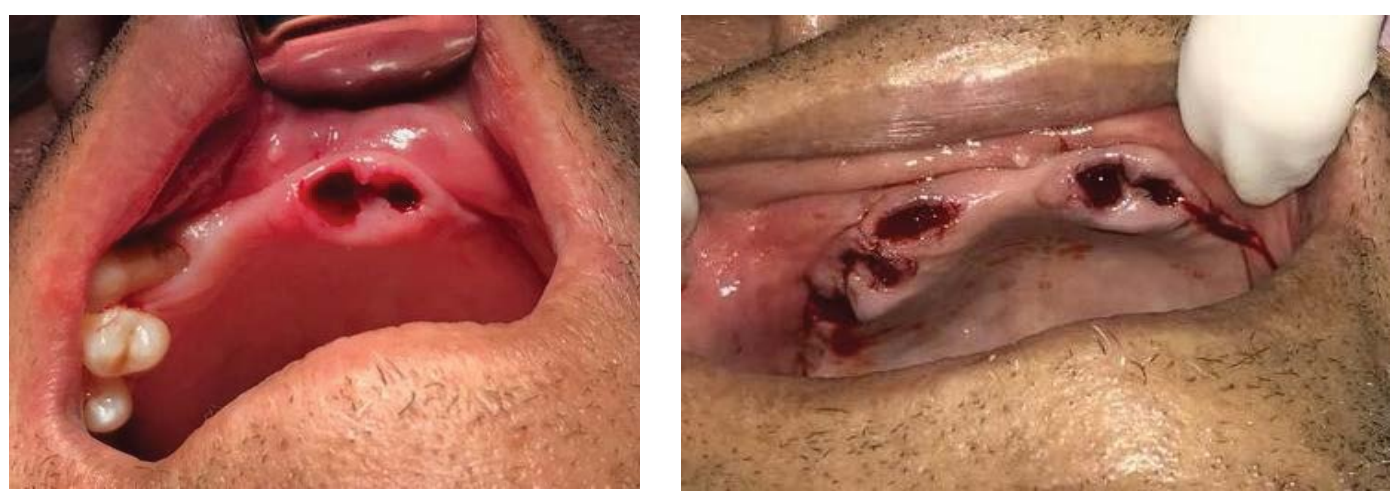

Figure 2: The surgical planning of the first day of surgery. 

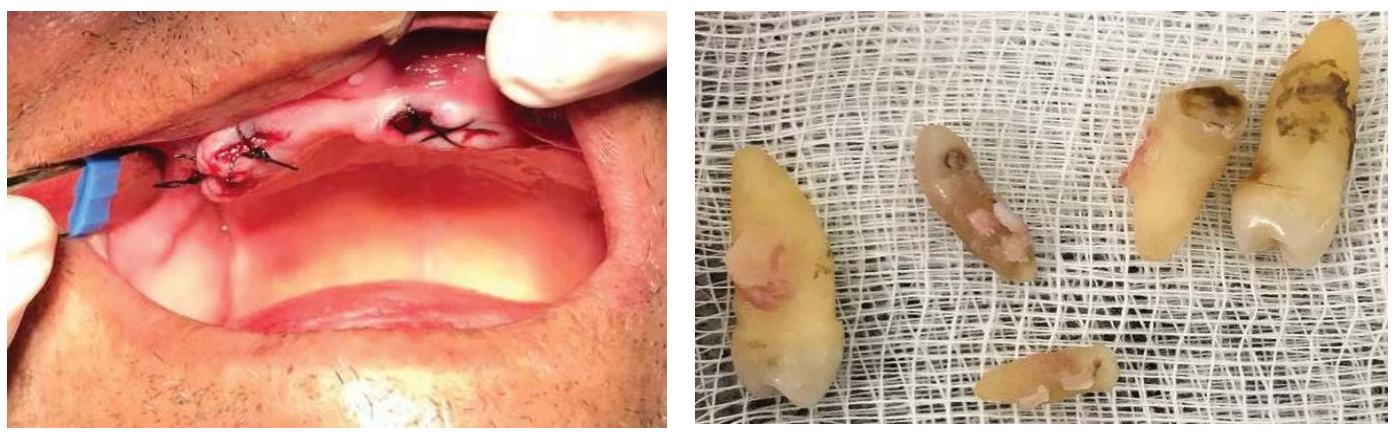

Figure 3: Cavity was cleaned with saline and curetted with Luca's curette.
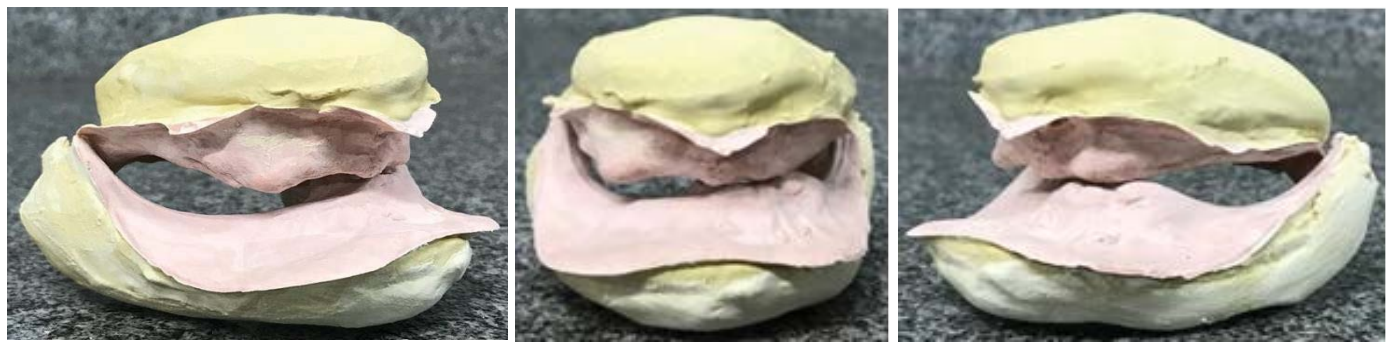

Figure 4: Acrylated prosthesis.
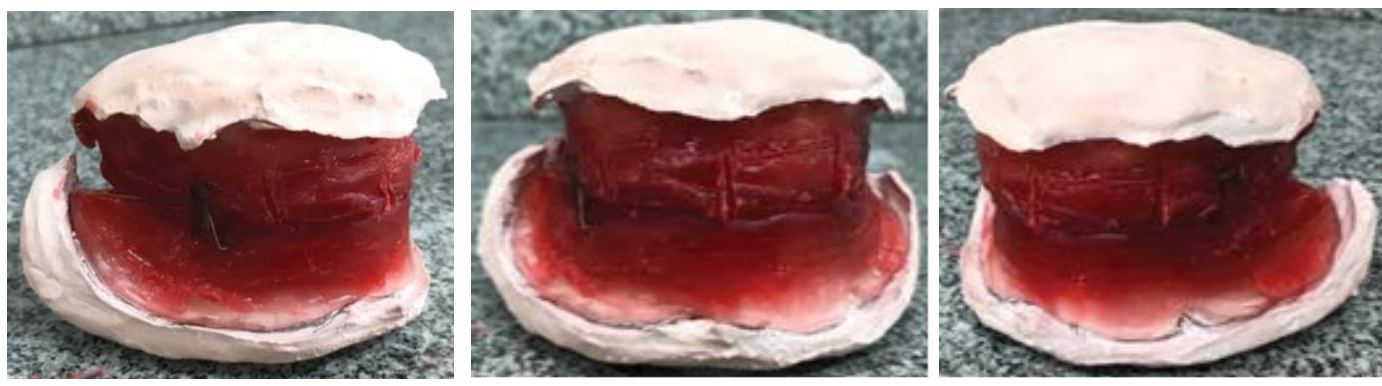

Figure 5: The prosthesis was filled with the wax rollers.

The suture was made with 3-0 silk thread and a sterile gauze was placed in the surgical area for hemostasis (Figure 3).

Because he was a risk patient, amoxicillin $500 \mathrm{mg}$ was prescribed to take one capsule every 8 (eight) hours for 5 (five) days and $500 \mathrm{mg}$ dipyrone to take one tablet every 6 (six) hours for 3 (three) days. The patient was guided on the postoperative and returned to the clinic for stitches withdrawal a week later.

Surgical planning for the second day of surgery was the extractions of the elements 31, 32, 33 and 34. For local infiltrative anesthesia, the anesthetic lidocaine $2 \%$. For mucoperiosteal detachment, the Molt detacher \#9 was used and the anchorage lever to dislodge the dental elements. For the teeth extractions, the forceps 151 was used.

Just as it was done in the other surgery, cleaning of the oral cavity with physiological saline solution was performed and curettage of the alveolus with Lucas curette. The silk thread 3-0 was used for suture and a sterile gauze was placed to perform local hemostasis. Unfortunately, there were no photos taken of the procedures. The same medications of the first surgery were prescribed for the patient. Postoperative guidelines were passed to the patient and, a week later, the stitches were removed. After this step, the patient returned for prosthesis evaluation within a month.

The patient was advised that, because of bone loss due to CKD, the prostheses would not be perfect, but they would be anchored in the arches with a prostheses gel holder. The patient returned to the clinic to start prosthetic part. For this, the anatomical and functional molding were made; the wax plane, prepared to obtain lines of reference (smile line, line canine, midline, Spee curve, buccal corridor, height of teeth); Proof of teeth and acrylated prosthesis (Figure 4).

After this step, the prosthesis was sent with the wax rollers to mark the reference lines and to adjust the occlusion. It was noticed, due to the chronic renal failure condition of the patient, that there was not enough retention or support points in the lower arch but had a good anchorage in the upper arch (Figure 5).

At the end of all these markings of reference lines, 

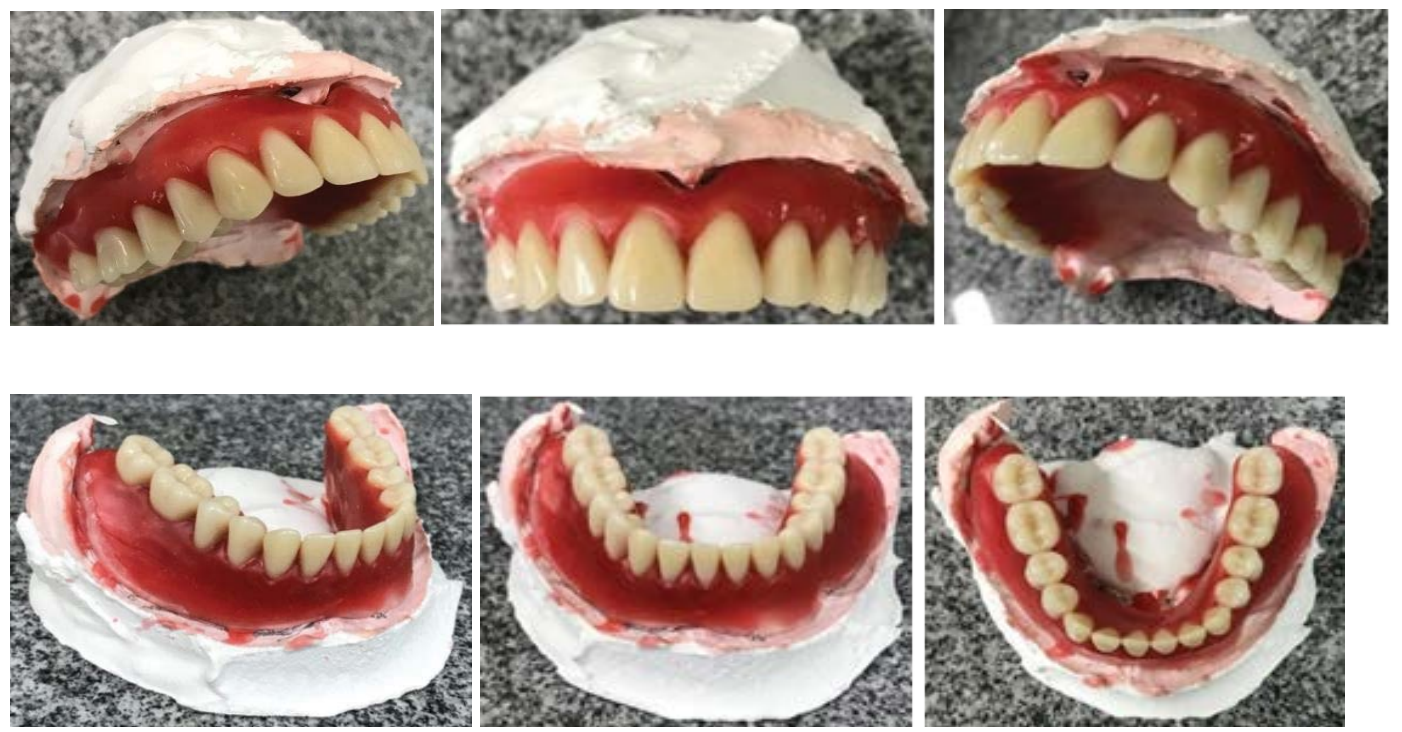

Figure 6: Stock teeth.

the prosthetist was asked to put the stock teeth into position. Then, it was possible to carry out the next step in the preparation of the prosthesis (Figure 6).

Next, the teeth were tested with the prosthesis in position in the patient's mouth. The occlusion of the same was observed and if all the vertical dimensions obtained in the previous steps were correct. After this stage, he was sent back to the prosthetist for the acrylization of the prostheses.

The patient was asked to wear a prosthesis gel holder, to return to the Clinic a week later and, within that time, to use the prosthesis. With less than a week, the patient returns to the clinic, saying he is not satisfied with the prosthesis, even with all the warnings that have been given since the beginning of the treatment.

Unfortunately, due to patient dissatisfaction, it was not possible to take pictures of the prostheses in position in both arches.

\section{Discussion}

According to Ruokonen [1], Chronic Kidney Disease (CKD) or his treatment may affect salivary flow rate and cause hyposalivation and thus increase the susceptibility to symptoms and discomfort, and the same author says the periodontal disease is a non-traditional risk factor for the CKD. It was noticed that the patient show signs of xerostomia and serious periodontal disease, and uremic breath.

It was noticed that, despite the patients having xerostomia, there was no increased risk of cervical caries. The loss of dental tissue due to caries is less prevalent in individuals with CKD than when compared to the general population due to nausea, esophageal regurgitation or induced vomiting (in cases of bulimia nervosa). According to Cholewa [4], although there are inconclusive outcomes regarding the prevalence of caries in hemodialysis patients, most studies describe a high incidence of periodontal disease in these patients, where poor oral hygiene with increased deposit of plaque and calculus, identifying the presence of gingival inflammation, deep periodontal pockets, clinical attachment level loss and bone loss. Those signs were found at the patient's clinical examination.

In the case of the patient of the report, there was no presence of caries in the dental elements, only wear on the buccal surfaces of the teeth 21 and 22, probably enamel fracture with dentin exposure, because of the masticatory load that was very high in these teeth.

One of the objectives of F.A.M.S. was to perform the dental treatment to extract the teeth and put upper and lower prostheses to prevent oral problems such as infections. According to Sturgil [7], in patients receiving hemodialysis, infective endocarditis can develop from the arteriovenous fistula. The American Heart Association (AHA) recommends antibiotic prophylaxis for patients undergoing hemodialysis who have known cardiac problems, which was not the case of the patient.

According to Medeiros [8], the blood pressure of the CKD patient should be monitored before and during the entire dental treatment. In the reported care, before the surgical procedure, the patient's pressure was checked and, when it was seen that it was within the standards for the surgery to be possible, the students obtained the approval of the teacher to begin.

Because of these bone losses, the patient's prosthesis was not sufficiently anchored to remain in the mouth. According to Genari Filho [9], a good retention (adhesion, cohesion, surface tension and atmospheric pressure), good support (bone with sufficient height and adhered fibromucosa) and a good stability were necessary for the prostheses to be anchored in the mouth (interdental relationship, ridge crest, base and adjacent musculature). 


\section{Conclusions}

It is important to maintain oral health in patients with CKD, since these are potential candidates for renal transplantation, and the transplantation will only be released if the patient is without infections, even without oral infections, since the immunosuppression that occurs after surgery.

As described in the case report, due to patient dissatisfaction when receiving the prosthetic parts, it is indispensable, in complex cases like this, the elaboration of a term of consent of the patient saying that the patient is aware of the situation and expectation of its treatment.

\section{References}

1. Ruokonen $H$, Nylund $K$, Meurman JH, Heikkinen AM, Furuholm J, et al. (2018) Oral symptoms and oral healthrelated quality of life in patients with chronic kidney disease from predialysis to posttransplantation. Clin Oral Investig.

2. Costa Filho JZ, Padilha WSM, Santos EKN (2007) Cuidados odontológicos em portadores de insuficiência renal crônica. Rev Cir Traumatol Buco Maxilo Fac 7: 19-28.
3. Nylund KM, Meurman JH, Heikkinen AM, Furuholm JO, Ortiz F, et al. (2017) Oral health in patients with renal disease: A longitudinal study from predialysis to kidney transplantation. Clin Oral Investig 22: 339-347.

4. Cholewa M, Madziarska K, Radwan-Oczko M (2018) The association between periodontal conditions, inflammation, nutritional status and calcium-phosphate metabolism disorders in hemodialysis patients. J App Oral Sci 26: e20170495.

5. Lopes LGS (2012) Atendimento odontológico ao paciente com insuficiência renal crônica em tratamento hemolítico: Recomendações para a prática clínica na esf.

6. Pupo MLMGS, Parizoto GA, Gonzaga CC, Lopes MGK (2010) Indice de risco odontológico para pacientes prétransplante renal submetidos à hemodiálise. Rev Sul-Bras Odontol 7: 50-56.

7. Sturgill J, Howell S, Perry MM, Kothari H (2016) Protocols for treating patients with end-stage renal disease: A survey of undergraduate dental programs. Special Care Dentist 36: $321-324$

8. Medeiros NH, Neves RRA, Amorim JNC, Mendonça SMS (2014) A insuficiência renal crônica e suas interferências no atendimento odontológico-revisão de literatura. Rev Odontol 26: 232-242.

9. Genari Filho H (2005) Requisitos funcionais e físicos em próteses totais. Revista Odontológica de Araçatuba 26: 36-43. 